\title{
ARTICLE Prefrontal-amygdala plasticity enabled by observational fear
}

\author{
Wataru Ito ${ }^{1}$ and Alexei Morozov $\mathbb{D}^{1,2,3}$
}

Observing fear in others (OF) is a form of social stress. In mice, it enhances inhibitory avoidance learning and causes the formation of silent synapses in the prefrontal-amygdala pathway. Here, we report that OF made that pathway prone to facilitation both ex vivo and in vivo. Ex vivo, OF enabled induction of long-term potentiation (LTP), expressed mostly postsynaptically and occluded by inhibitory avoidance training. In vivo, OF enabled facilitation of the dmPFC-BLA pathway by inhibitory avoidance training. The facilitation persisted during the first $4 \mathrm{~h}$ after the training when the prefrontal cortex and amygdala are involved in memory consolidation. Thus, the OF-generated silent synapses likely enable plasticity that may enhance the consolidation of inhibitory avoidance memories.

Neuropsychopharmacology (2019) 44:1778-1787; https://doi.org/10.1038/s41386-019-0342-7

\section{INTRODUCTION}

Observing others in fear or pain is a form of psychosocial stress, which, in humans, can lead to PTSD [1, 2]. The relevant behavioral effects in rodents are obtained by exposure to distressed conspecific. They include the formation of contextual and cued fear memories [3-5], the enhancement of performance in the twoway avoidance and contextual fear learning tasks given immediately after the exposure [6], and the development of anhedonia and anxiety-like behaviors [7, 8]. While the underlying mechanisms of these effects are not well understood, recent findings have implicated distinct microcircuits in the anterior cingulate cortex (ACC) [9], a subdivision of the dorsomedial prefrontal cortex (dmPFC), and its BLA-projecting neurons [10] in the observational fear (OF) learning.

Recently, we have found that the OF procedure, in which the subject mouse is exposed to a conspecific receiving electrical footshock, enhanced inhibitory avoidance (IA) learning. At the same time, the proportion of silent synapses, which only contain the NMDA receptor, increased in the input from the dorsomedial prefrontal cortex (dmPFC) to the basolateral amygdala (BLA). Interestingly, subanesthetic ketamine, administered immediately after OF, abolished both effects [11]. Meanwhile, experiments with pharmacological manipulations have provided evidence that (a) dmPFC is critical for IA learning [12, 13], (b) BLA plays a modulatory role $[14,15]$, and (c) strengthening of functional interaction between dmPFC and BLA immediately after IA training enhances IA memory consolidation $[16,17]$. It raises a question whether the OF-generated silent synapses participate in the OFenhanced IA learning.

Because silent synapses can incorporate AMPAR and convert to functional synapses [18-23], such de novo generation of silent synapses in adults may contribute to the aberrant synaptic reorganization as seen in drug addiction and after brain injuries [18]. Therefore, we hypothesized that IA training causes the conversion of the OF-generated silent synapses to facilitate the dmPFC-BLA synaptic transmission during IA learning.
The hypothesis was tested by quantifying plasticity and silent synapses along the OF-IA paradigm ex vivo. Furthermore, to obtain direct evidence of synaptic facilitation in the dmPFC-BLA synapses during IA training, we recorded light-evoked local field potentials (LFPs) in BLA by stimulating the dmPFC afferents in vivo. The experiment revealed that OF enabled facilitation of dmPFC-BLA pathway by IA training presumably via unsilencing of the OF-generated silent synapses.

\section{MATERIALS AND METHODS}

Animals

129SvEv/C57BL/6 N F1 hybrid male mice were produced from breeding trios of one C57BL/6 N male and two 129SvEv females, weaned at p21-25, and housed two littermates per cage as described [11]. Channelrhodopsin-2 expressing adeno-associated virus (AAV-ChR2) was injected at p28, and experiments were performed at p60-75, according to Virginia Tech IACUC-approved protocol.

\section{Behavioral treatments}

Observational fear. The OF procedure [3] was performed in a fear conditioning chamber (Med Associates, St. Albans, VT), divided into two unequal compartments by a transparent Plexiglas wall with $7 \mathrm{~mm}$ diameter holes, spaced at 2-cm interval, to allow auditory and olfactory cues, and whisker to whisker interaction. In the larger $26 \times 20 \times 26 \mathrm{~cm}$ compartment, a stainless steel rod floor was covered with a white plastic sheet. In the smaller $26 \times 9 \times 26$ $\mathrm{cm}$ compartment, the rod floor was exposed. Cagemates observer and demonstrator were placed, respectively, in the larger and smaller compartments. After 5 min acclimation, 24 footshocks $(1 \mathrm{~mA}, 2 \mathrm{~s}$, every $10 \mathrm{~s})$ were delivered to the demonstrator. The observer was returned to the home cage and housed alone. In the OF control procedure (denoted as "OF cont"), all the manipulations were the same except demonstrators did not receive footshocks.

${ }^{1}$ Virginia Tech Carilion Research Institute, Roanoke, Virginia, USA; ${ }^{2}$ School of Biomedical Engineering and Sciences, Virginia Tech, Blacksburg, Virginia, USA and ${ }^{3}$ Department of Psychiatry and Behavioral Medicine, Virginia Tech Carilion School of Medicine, Roanoke, Virginia, USA

Correspondence: Wataru Ito (wataru.ito@gmail.com) or Alexei Morozov (alexeim@vtc.vt.edu)

Received: 7 October 2018 Revised: 7 January 2019 Accepted: 6 February 2019

Published online: 13 February 2019 
Inhibitory avoidance. The one trial step-through paradigm [24] was done in the Gemini Avoidance System with the Gemini Mouse Start Box (San Diego Instruments, San Diego, CA). For training, the mouse was placed in the starting side of the chamber with a guillotine door closed, and lights turned off. After $60 \mathrm{~s}$ acclimation, the chamber- and cue- lamps were turned on in the starting side, and the door was opened. Once mice entered the dark side, the door was closed, and a $0.15 \mathrm{~mA} 1 \mathrm{sec}$ electrical footshock was given to the IA training group (denoted as " $I A$ ") or not given to the IA training control group (denoted as "I $\left.A_{\text {cont }}{ }^{\prime \prime}\right)$. Mice were returned to the home cages $30 \mathrm{~s}$ after the guillotine door closure.

\section{Surgery}

ChR2-AAV pseudo-type 1 virus at the titer of $10^{12}$ viral particles/ml was prepared by University of North Carolina Gene Therapy Vector Core (Chapel Hill, NC) using Addgene plasmid 20071 [25], in which ChR2-Venus expression is driven by CAG promoter. P28 mice were anesthetized with Ketamine/ Xylazine/Acepromazine $(100 / 5.4 / 1 \mathrm{mg} / \mathrm{kg})$ injected intramuscularly and placed in a stereotaxic apparatus (David Kopf, Tujunga, CA). After minimum craniotomy ( 0.5 mm diameters), preserving the dura mater, a heater-pulled glass pipette (shaft: 0.6/0.4 mm external/internal diameter, tip: $50 \mu \mathrm{m}$, Drummond, Broomall, PA) filled with the virus solution was slowly lowered to the target brain areas. A volume of $0.5 \mu \mathrm{l}$ of the solution were injected bilaterally, using a syringe pump connected to the pipette through plastic tubing filled with water, in dmPFC at $1.3 \mathrm{~mm}$ anterior, $0.4 \mathrm{~mm}$ lateral from bregma, and $1.3 \mathrm{~mm}$ ventral from brain surface as described [26]. For in vivo recordings, mice at p60 were anesthetized with Ketamine/ Xylazine/Acepromazine $(100 / 5.4 / 1 \mathrm{mg} / \mathrm{kg})$ injected intramuscularly and positioned in a stereotaxic apparatus (David Kopf, Tujunga, CA). Four stainless steel bearing balls $(0.5 \mathrm{~mm}$ diameter) with silver wires were embedded in small holes at the two frontal bones and the left and right parietal bones at the border with the interparietal bones for electrical ground/ reference electrodes. Custom-made optrodes were fabricated with a miniature LED (EZ500-n, $460 \mathrm{~nm}$, CREE), coupled to an optical fiber (0.66 NA, $0.2 \mathrm{~mm}$ core diameter, Prizmatix) with UV-cured index matched glue (Norland Optical Adhesive 85, Norland) and two $33 \mu \mathrm{m}$ tungsten wires (polyimide-insulated, California Fine Wire) extended $0.5 \mathrm{~mm}$ beyond the optical fiber. The optrodes were implanted bilaterally, so that the tips of the recording wires were targeted inside $\mathrm{BLA}$ ( AP $-1.5, \mathrm{ML} \pm 3.1$ from bregma and DV -4.2 from brain surface), and the tip of the optical fiber remained above BLA. The optrodes were affixed to the skull with cyanoacrylate (IC-2000TM Rubbertoughened CA, Bob Smith Industries) and embedded in dental acrylic. For post-operation analgesia, ketoprofen $(5 \mathrm{mg} / \mathrm{kg})$ was administered subcutaneously.

\section{Ex vivo electrophysiology}

General. Amygdala slices, $300 \mu \mathrm{m}$ thick, were prepared as described [26] using ice-cold partial sucrose artificial cerebrospinal fluid (ACSF) solution containing (in mM) $80 \mathrm{NaCl}, 3.5 \mathrm{KCl}, 4.5$ $\mathrm{MgSO}_{4}, 0.5 \mathrm{CaCl}_{2}, 1.25 \mathrm{H}_{2} \mathrm{PO}_{4}, 25 \mathrm{NaHCO}_{3}, 10$ glucose, and 90 sucrose equilibrated with $95 \% \mathrm{O}_{2} / 5 \% \mathrm{CO}_{2}$ [27], incubated at 33 ${ }^{\circ} \mathrm{C}$ for $30 \mathrm{~min}$ and stored in the same solution at room temperature for at least $45 \mathrm{~min}$ before recording. Recording chamber was superfused at $2 \mathrm{ml} / \mathrm{min}$ with ACSF equilibrated with $95 \% \mathrm{O}_{2} / 5 \%$ $\mathrm{CO}_{2}$ and containing (in mM) $119 \mathrm{NaCl}, 2.5 \mathrm{KCl}, 1 \mathrm{MgSO}_{4}, 2.5 \mathrm{CaCl}_{2}$, $1.25 \mathrm{H}_{2} \mathrm{PO}_{4}, 26 \mathrm{NaHCO}_{3}, 10$ glucose, and $100 \mu \mathrm{M}$ picrotoxin $(\mathrm{pH}$ 7.4). Whole-cell recordings were obtained at $30 \pm 1{ }^{\circ} \mathrm{C}$ with EPC-10 amplifier and Pulse v8.76 software (HEKA Elektronik, Lambrecht/ Pfalz, Germany). Putative glutamatergic neurons in BLA were identified by their pyramidal morphology [28] under Dodt gradient contrast optics (custom made) at $850 \mathrm{~nm}$ LED illumination (Thorlabs, Newton, $\mathrm{NJ}$ ) and were recorded using 2-4 M $\Omega$ pipette filled with (in $\mathrm{mM}$ ) $120 \mathrm{~K}$-gluconate, $5 \mathrm{NaCl}, 1 \mathrm{MgCl}_{2}, 10$ HEPES, 0.2 EGTA, 2 ATP-Mg, and 0.1 GTP-Na for current-clamp experiments, or with $120 \mathrm{Cs}$-methanesulfonate, $5 \mathrm{NaCl}, 1 \mathrm{MgCl}_{2}$, 10 HEPES, 0.2 EGTA, 2 ATP-Mg, 0.1 GTP-Na, and $10 \mathrm{mM}$ QX314 for voltage-clamp experiments, with pH of 7.3 and osmolarity of 285 Osm. Membrane potentials were corrected by the junction potential of $12 \mathrm{mV}$. Series resistance (Rs) was $10-20 \mathrm{M} \Omega$ and monitored throughout experiments. Data were not included in the analysis if Rs changed more than $20 \% .470 \mathrm{~nm} 1 \mathrm{~ms}$ light pulses were generated using an LED lamp (Thorlabs) and a custom LED driver based on MOSFET and were delivered through a $\times 40$ objective lens (Olympus, Center Valley, PA) at 0.3 to $2.5 \mathrm{~mW}$, calibrated by a photodiode power sensor (Thorlabs) at the tip of the lens.

LTP induction. STDP-LTP experiments were performed as described in [26]. In most LTP experiments, the single test pulses were given every $20 \mathrm{~s}$. In the subset of slices, in which the paired pulses ( $50 \mathrm{~ms}$ interval) were used as test pulses for measuring paired-pulse ratio, they were given every $40 \mathrm{~s}$ instead of $20 \mathrm{~s}$, to keep the number of test pulses the same as in other LTP experiments.

Induction of plasticity in the voltage-clamp and measuring AMPAR/ NMDAR ratio. To induce plasticity, eighty pulses of the $2 \mathrm{~Hz}$ presynaptic stimulation were paired with a steady postsynaptic depolarization to $0 \mathrm{mV}$ [29]. AMPAR currents were recorded from BLA neurons held at $-75 \mathrm{mV}$ during the $5 \mathrm{~min}$ before and $10 \mathrm{~min}$ after the induction of plasticity. They were evoked by the light pulses, adjusted to obtain baseline responses within the range of -50 to $-200 \mathrm{pA}$ and delivered every $20 \mathrm{~s}$. The mixed AMPAR + NMDAR currents were recorded from cells held at $+40 \mathrm{mV}$ immediately before induction of plasticity and immediately after the 10 min recording of the AMPAR currents. The mixed currents were evoked by 5 light pulses given at the $5 \mathrm{~s}$ interval. The AMPAR currents were measured at the peak response. The NMDAR currents were measured at $50 \mathrm{~ms}$ after the peak of the mixed current. The AMPAR/NMDAR indices were obtained from the NMDAR currents and the AMPAR currents during the baseline and during the minutes 9-10 after induction of plasticity.

Silent synapses were quantified by the minimal-like stimulation technique [30-32] using the $25 \mu \mathrm{m}$ diameter light spot as described in [11]. AMPAR EPSC were recorded at $-75 \mathrm{mV}$ and mixed AMPAR/NMDAR EPSC at $+40 \mathrm{mV}$. Stimulus intensity was adjusted to obtain the success rate for AMPAR EPSC within the range of $30-70 \%$. No threshold or amplitude criteria of minimal stimulation were applied. Twenty-five to hundred traces were recorded at each membrane potential. Failures and responses at $-75 \mathrm{mV}\left(\mathrm{F}_{-75}\right)$ and at $+40 \mathrm{mV}\left(\mathrm{F}_{+40}\right)$ were identified by visual analysis of traces. The fraction of silent synapses was calculated using an equation: 1 - $\operatorname{Ln}\left(\mathrm{F}_{-75}\right) / \operatorname{Ln}\left(\mathrm{F}_{+40}\right)$ [33].

In vivo recordings

One mouse of each littermate pair was transduced with AAV-ChR2 bilaterally in dmPFC and implanted with the optrodes bilaterally in BLA, as described above. At p70-90, LFPs were evoked in BLA by blue light stimulation of dmPFC terminals expressing ChR2 and recorded from the subject mouse, singly housed in the home cage without the cage lid. LFPs were recorded using the RHA2000Series Amplifier USB Evaluation Board (RHA2000-EVAL, Intan Technologies). The blue light pulses $(0.5 \mathrm{~ms}, 2-3 \mathrm{~mW}$ at the tip of optrode) were adjusted to obtain the fEPSP slope at $30-40 \%$ of the maximum. The LED driver (PlexBright LD-1, Plexon) was analog-modulated by DAQ (Analog Shield, Digilent). The LED driving current was routed to the optrodes in either hemisphere by electrical relays (Arduino 4 relays shield, Arduino). Arduino with a custom Arduino sketch controlled both the DAQ and the relays to give the light stimulation on each side every $30 \mathrm{~s}$, alternating 
A

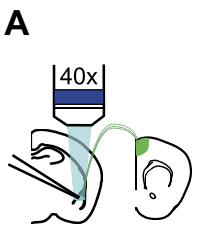

c

\section{B}

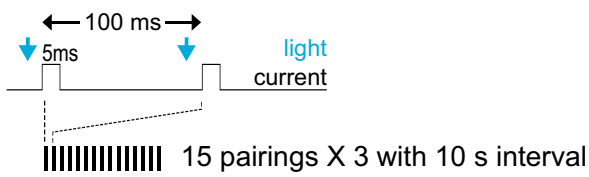

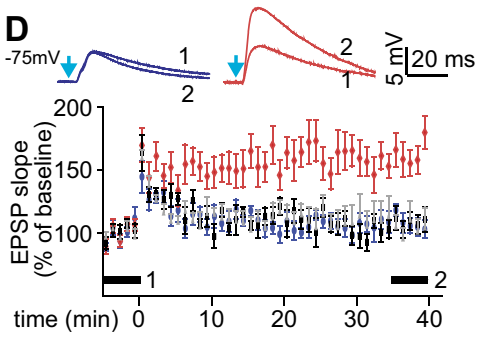

$-75 \mathrm{mv}+\sqrt{1} \pm \sqrt{\frac{2}{1}} \vec{E}_{\mid 20} \mid 20 \mathrm{~ms}$

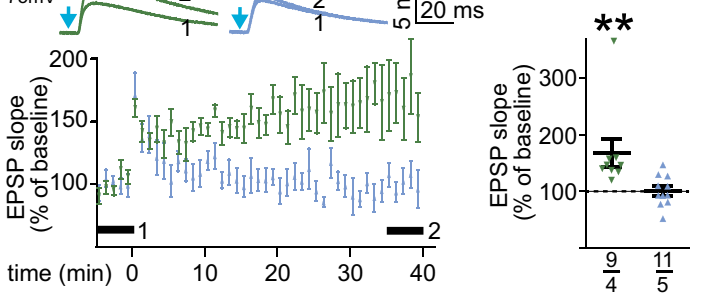

Fig. 1 OF enables induction of LTP, but IA training occludes it. a Recording scheme. b LTP induction protocol. c Timelines for 6 groups of mice. d LTP in dmPFC-BLA pathway. Each dot represents data averaged over $1 \mathrm{~min}$. Insets represent examples of averaged EPSPs before (1) and after (2) induction as indicated by thick bars. Right, EPSP slopes for individual cells and their averages at (2). The numbers of cells (upper) and animals (lower) are shown under each plot. Each group is color coded as in (c). Error bars represent SEMs, ${ }^{* *} p<0.01,{ }^{* * *} p<0.001$, compared to baseline, Wilcoxon signed rank test

the sides every $15 \mathrm{~s}$. Data were processed using custom scripts written in MATLAB (MathWorks) and Clampfit software (Molecular Devices). The observer mice were habituated to the recording environment by connecting to the recording system for $2-3 \mathrm{~h}$ per day during 2-3 consecutive days. Once the evoked LFPs stabilized, on the next day (Day 1), mice underwent OF and then IA training $24 \mathrm{~h}$ later (Day 2). On Day 1, after baseline stabilized, the animal was transferred gently, without disconnecting the recording tether, into the OF chamber. Immediately after OF, the animal was returned to the home cage, and recording continued. On Day 2, after baseline stabilization, the animal was gently disconnected from the recording tether, because the IA chamber was not compatible with recording, and subjected to IA training. Immediately after IA training, the animal was placed back in the home cage and reconnected to continue recording. Recording continued for up to $8 \mathrm{~h}$ after each manipulation. The positions of optrodes were confirmed by post hoc histological analysis.

\section{Data Analysis}

In all ex vivo experiments, behavioral training was done by $\mathrm{WI}$ and the whole-cell recordings-by $\mathrm{AM}$ - to ensure blinding. For the in vivo studies, behavioral training and recording were done by the same person (Wl), because these procedures cannot be separated. However, the statistical analysis was done by AM. Statistical analyses were performed using GraphPad Prism 5 (GraphPad Software, La Jolla, CA). Normality was tested using the Shapiro-Wilk test. Datasets with normal distribution were compared using the one sample $t$ test, the two-tale unpaired or paired $t$-test as indicated. The datasets with non-normal distribution were compared using the Mann-Whitney test, the Wilcoxon matched pairs test, and the Wilcoxon signed rank test. The difference was deemed significant with $p<0.05$. The two-tailed $p$-value was calculated for correlation analyses.

\section{RESULTS}

OF enables facilitation of dmPFC-BLA pathway ex vivo whereas IA training reverses this effect

The OF paradigm (OF) generates silent synapses in the dmPFCBLA pathway of the observer's brain and facilitates subsequent inhibitory avoidance learning (IA) [11]. To study possible involvement of the OF-generated silent synapses in IA, we first tested whether OF and IA training influenced synaptic plasticity in the dmPFC-BLA pathway. Using brain slices prepared from mice transduced with AAV-ChR2 in the dmPFC (Fig. S1), the synaptic responses in dmPFC-BLA pathway were evoked by blue light stimulation of the dmPFC axons in BLA and recorded from BLA principal neurons (Fig. 1a). Synaptic plasticity was tested by a modified spike-timing-dependent plasticity (STDP) protocol [34], in which the onset of excitatory postsynaptic potential (EPSP) preceded action potential (AP) by $3-5 \mathrm{~ms}$ as described in [26]. In that earlier study, the EPSP-AP pairing, repeated 75 times, induced LTP in the dmPFC-BLA synapses [26]. Here, to detect possible enhancement of LTP by OF, the number of the EPSP-AP repetitions was reduced to 45 (Fig. 1b), which was below the threshold for LTP induction in control mice. Amygdala slices were prepared from 6 groups of mice. On Day 1, the animals underwent either the OF ("OF") or the OF control ("OF $\left.{ }_{\text {cont }}{ }^{\prime \prime}\right)$ procedure and then underwent, on Day2, either the IA training ("IA") or the IA training control ("I $\mathrm{A}_{\text {cont }}$ ") procedure. Slice preparation started by sacrificing the animals either 2 or $18 \mathrm{~h}$ later (Fig. 1c). LTP data are shown in Fig. 1d. LTP was observed in the "OF-IA $\mathrm{A}_{\text {cont }}$ " group, but

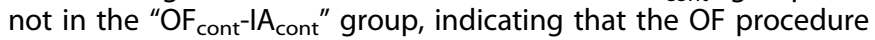
enabled LTP. Meanwhile, the "OF-IA" group showed no LTP, indicating that IA training abolished the OF-enabled LTP. There was no difference between the "OF ${ }_{\text {cont }}-\mathrm{IA}_{\text {cont }}$ " and "OF ${ }_{\text {cont }}-\mathrm{IA}$ " groups, indicating that IA training per se did not enable depressing responses to the LTP induction protocol. The 
OF-enabled and IA-training-abolished plasticity were also tested $18 \mathrm{~h}$ after the IA/ IA $A_{\text {cont }}$ procedure. The "OF-IA $\mathrm{A}_{\text {cont }}-18 \mathrm{~h}$ " group showed LTP, indicating that the OF-enabled plasticity persisted for about 2 days after the OF procedure, whereas the "OF-IA-18h" group showed no LTP, indicating that the plasticity did not return once eliminated by IA training.

\section{OF enables postsynaptic mechanisms of plasticity}

Several studies provided evidence that LTP in the cortical inputs to the lateral amygdala is expressed presynaptically [35-41]. To determine the expression site of the OF-enabled LTP observed in the OF-IA $A_{\text {cont }}$ group, at first, the paired-pulse ratio (PPR), an indicator of a change in the presynaptic release probability [42], was analyzed before and after the LTP induction. The effect of LTP induction on PPR varied among cells, and there was a tendency towards a decrease, but it did not reach a statistical significance (Fig. 2a, b). Second, we tested two indicators of presynaptic LTP [29], the correlation between LTP and relative $\mathrm{CV}^{-2}$ (CV stands for coefficient of variation of the EPSP slope) (Fig. 2C) and the changes in the CV (Fig. 2d), and found no significance in either. One interpretation of these results is that the presynaptic compartment is not the major site for the LTP expression, which could be a characteristic feature of the OF-enabled LTP. Alternatively, ChR2, known to increase the neurotransmitter release probability [43], may abolish presynaptic plasticity via the ceiling effect. In the case of the artifact from ChR2, the LTP induced in naive mice would not express presynaptically either. We tested this by using a stronger STDP protocol with 75 EPSP-spike pairings [26]. It produced LTP of a similar magnitude with the OF-enabled LTP $(149 \pm 10.3 \%, n=18$, $p<0.001$ ) (Fig. 2e). However, the decreases in PPR (Fig. 2f), the correlation between LTP and relative $\mathrm{CV}^{-2}$ (Fig. 2g), and the decreases in CV (Fig. 2h) were significant, indicating presynaptic LTP expression. Thus, the presence of ChR2 per se does not abolish the presynaptically expressed LTP.

The lack of (a) significant changes in PPR after LTP induction, (b) correlation between LTP and relative $\mathrm{CV}^{-2}$, or (c) changes in CV after LTP, suggest that the OF-enabled LTP is expressed postsynaptically and increases the ratio between postsynaptic currents mediated by AMPA and NMDA receptors. Measuring this ratio requires voltage-clamp recordings and $\mathrm{Cs}+$ in the internal solution, which are incompatible with our STDP protocol. As an alternative, we induced plasticity by pairing 80 pulses of the $2 \mathrm{~Hz}$ presynaptic stimulation with a steady postsynaptic depolarization to $0 \mathrm{mV}$ [29] and measured the AMPA/NMDA ratio before and 10 min after the induction. The short $10 \mathrm{~min}$ time was chosen because of poor stability of whole-cell patch after alternating membrane potential between $-75 \mathrm{mV}$ and $+40 \mathrm{mV}$ for the AMPAR and mixed AMPAR + NMDAR EPSCs, respectively. The pairing protocol caused significant increases in the AMPAR EPSCs in mice that underwent OF (OF-IA $A_{\text {cont }}$ on Fig. $\left.2 \mathrm{i}-\mathrm{I}\right)$, but not in the controls $\left(\mathrm{OF}_{\text {cont }}-\mathrm{IA}_{\text {cont }}\right)$ or in mice that underwent $\mathrm{OF}$ followed by IA training (OF-IA). The pairing protocol did not change the NMDAR EPSCs in all three groups (Fig. $2 \mathrm{~m}$ ) but increased the AMPAR/NMDAR EPSCs ratio in the OF-IA cont group (Fig. 2n). These data indicate that $\mathrm{OF}$ enables the postsynaptically expressed plasticity.

The OF-generated silent synapses require hours to form, persist for two days, and are eliminated by IA training

The postsynaptic expression of the OF-enabled LTP could be mediated by unsilencing of the OF-generated silent synapses that appear in the dmPFC-BLA pathway one day after OF [11]. Here, we quantified silent synapses at different time points after OF procedure, ranging from $4 \mathrm{~h}$ to one week, and after OF was followed by IA training (Fig. 3a). Silent synapses were assessed by giving minimal-like stimulation of the dmPFC axons and then quantifying failure rates of the postsynaptic responses recorded at $-75 \mathrm{mV}$, which are mediated by AMPA receptors, and responses recorded at $+40 \mathrm{mV}$, mediated by both AMPA and NMDA receptors (detailed in Materials and methods). The nature of the responses recorded at -75 and $+40 \mathrm{mV}$ was confirmed pharmacologically by recordings in the presence of an AMPA receptor blocker CNQX and an NMDA receptor blocker APV (Fig. S2).

The proportion of silent synapses in the OF-treated mice remained higher than in controls at $42 \mathrm{~h}$ after OF $(p<0.05$, unpaired $t$-test, compared to the OF control), but not at $4 \mathrm{~h}$ or one week after OF ( $p>0.05)$ (Fig. 3b-f, i). In the mice that underwent IA training after $\mathrm{OF}$, the proportion of silent synapses was no longer different from that in the OF controls both at 2 and $18 \mathrm{~h}$ after IA training $(p>0.05)$ (Fig. $3 g-i)$. It indicated that silent synapses were generated in a slow process, which required more than $4 \mathrm{~h}$, and remained for two days, at least, but no longer than one week. IA training, however, removed the silent synapses rapidly and they did not reappear $18 \mathrm{~h}$ later. Those observations led to a prediction that IA training facilitated the dmPFC-BLA pathway by unsilencing the OF-generated silent synapses.

Observing fear enables facilitation of dmPFC-BLA pathway by IA training in free moving mice

To examine how OF and IA training affected the dmPFC-BLA pathway in vivo, we recorded LFPs evoked in BLA by blue light stimulation of dmPFC axons expressing ChR2 in awake free moving mice. The mice, transduced with AAV-ChR2 in dmPFC and implanted with optrodes in BLA (Fig. 4a), underwent OF or the OF control procedure and then were trained in IA on the next day. The evoked LFPs were recorded before and after OF and IA training from the animals in their top-open home cages without the cagemates. Mice were first habituated to the recording environment for 2-3 h per day during 2-3 consecutive days until the evoked-LFP responses stabilized. For the OF and IA training, the animals were transferred from the home cage to the corresponding training chamber. Handling the animal typically caused transient depression of evoked LFPs, which lasted for 30-60 min (data not shown). Similar depression was observed after OF and IA training (Figs. 4 and 5). For IA training, because of incompatibility of the training chamber with recording in vivo, the subjects required disconnection and reconnection to the recording system, and LFPs were not recorded for about 10 min during the training. After OF training, there were no differences between the dynamics of the evoked LFP between the OF and OF control groups and LFPs returned to the baseline within $1 \mathrm{~h}$ after training (both groups, during $1-2 \mathrm{~h}$ after OF training, normalized to the baseline fEPSP amplitude: $p>0.05$, normalized fEPSP slope: $p>$ 0.05 , Wilcoxon signed rank test) (Fig. 4b, c). It indicated that OF training did not change the dmPFC-BLA synaptic efficacy.

In contrast, the OF and OF control groups showed different fEPSCs dynamics after IA training. In the OF group, LFP did not depress when animals were reconnected, and then, showed significant increases in EPSP amplitudes and slopes, which lasted for about $4 \mathrm{~h}$ (Fig. 5). In the OF control group, there was no facilitation of LFPs. It indicated that OF enabled a transient in vivo facilitation of the synaptic transmission between dmPFC and BLA by IA training.

\section{DISCUSSION}

The main findings of this study are that observing fear in others enables facilitation of the dmPFC-BLA pathway both ex vivo by a weaker LTP protocol, and in vivo by IA training, and that the presence of the OF-generated silent synapses coincides with the plasticity. Therefore, unsilencing of the OF-generated silent synapses is likely responsible for that new plasticity ex vivo and presumably in vivo.

For testing LTP ex vivo, we used an STDP induction protocol, which involves co-incidental presynaptic stimulation and postsynaptic spiking and may represent a process relevant to 
A
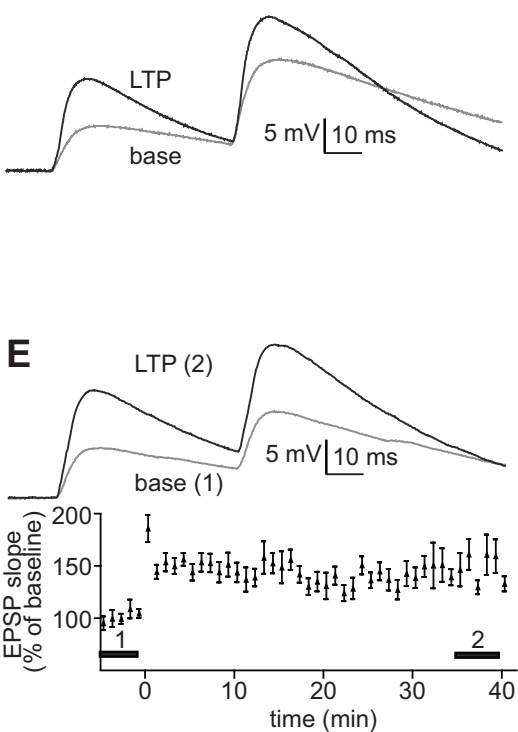

I

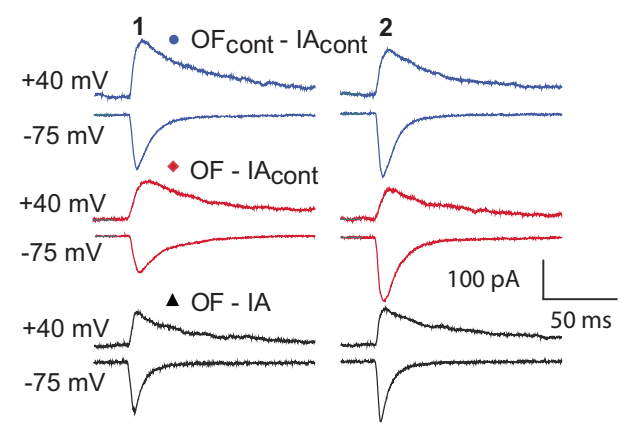

B

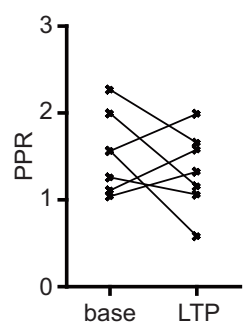

$\mathbf{F}$

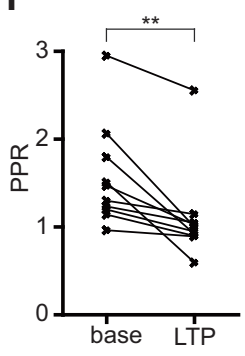

C

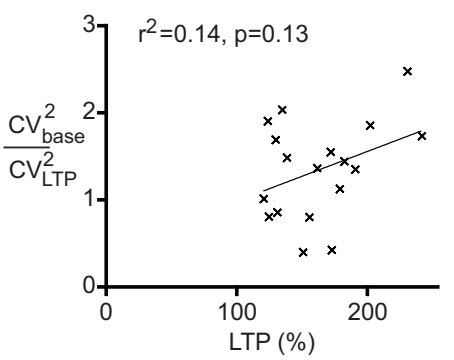

G

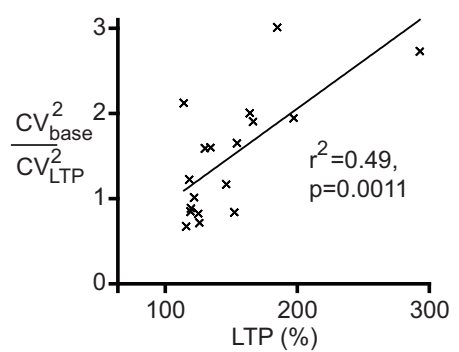

D

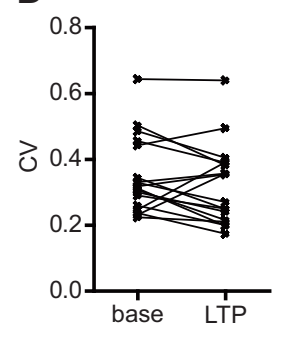

H

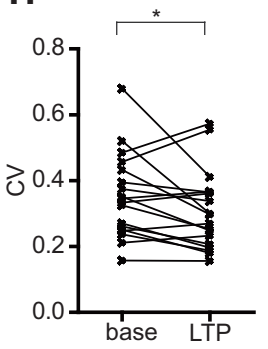

$\mathbf{J}$

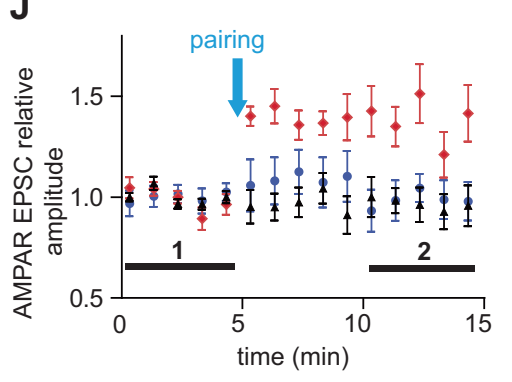

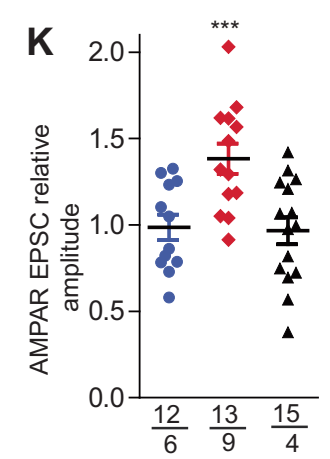

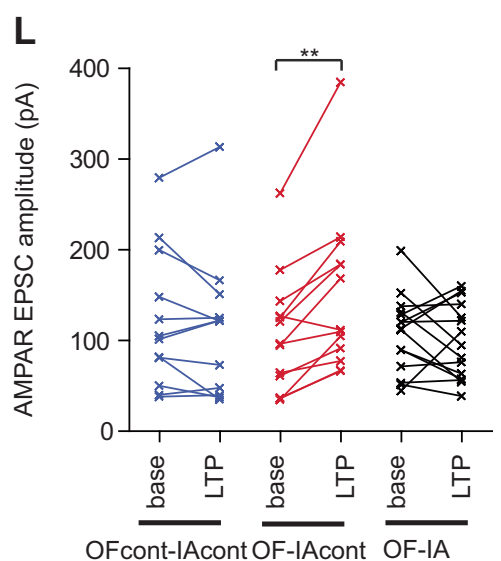

M
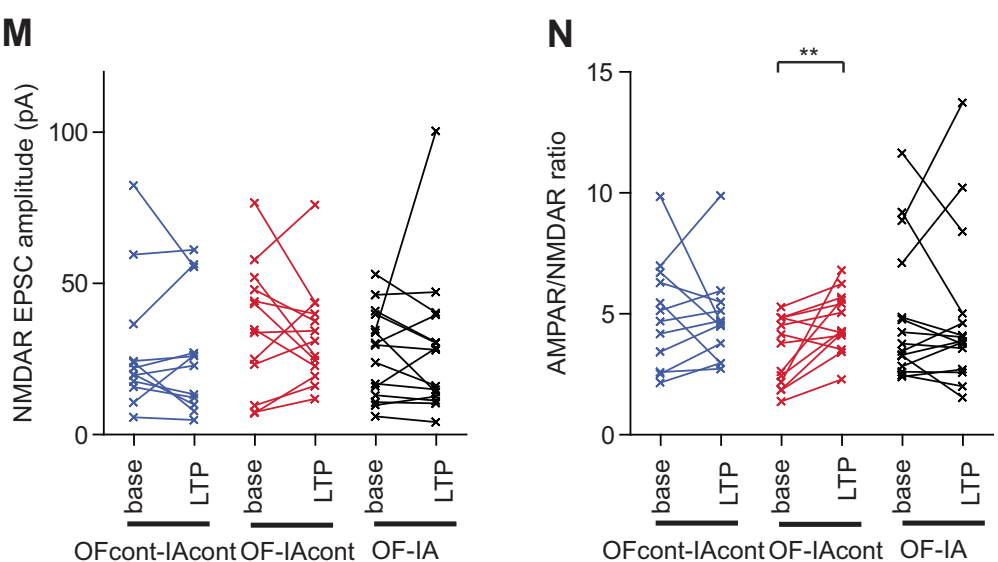

Fig. 2 OF enables postsynaptically expressed plasticity. a-d Indices of presynaptic plasticity in LTP experiments using the weak STDP protocol in OF-IA $A_{\text {cont }}$ group. a Examples of paired-pulse recording before (gray, average of the min -5 to -1 of baseline) and after LTP induction (black, average of the min 35-40 after induction). b Summary data for paired-pulse ratios. Lines connect data points representing the same neuron. c Relative $\mathrm{CV}^{-2}$ as a function of LTP $(n=18)$. d CV at baseline and during the minutes 35-40 after LTP induction. e-h The same comparisons using the strong STDP protocol in OF-naive group. e Upper: An example of paired-pulse recording before (1: gray) and after (2: black) LTP induction. Lower: LTP data $(n=18)$. f Summary data for paired-pulse ratios $(n=10)$. g Relative $\mathrm{CV}^{-2}$ as a function of LTP $(n=18)$. h CV at baseline and during the minutes 35-40 after LTP induction. i-n OF-enabled plasticity induced by the pairing protocol involves increases in the AMPAR current, but not NMDAR current. (i) Examples of AMPAR (lower) and AMPAR/NMDAR mixed currents (upper) before (1) and after (2) the pairing in neurons from $\mathrm{OF}_{\text {cont }}-\mathrm{IA}_{\text {cont }}$ (blue), OF-IA $\mathrm{A}_{\text {cont }}(\mathrm{red})$, and OF-IA (black) groups. Averages of 5 consecutive sweeps are shown. $\mathbf{j}$ LTP data for the AMPAR EPSC amplitudes before and after the pairing. Each point represents data for $1 \mathrm{~min}$. $\mathbf{k}$ AMPAR EPSC amplitudes during the last 5 sweeps of recording, normalized to the mean baseline amplitudes. Values for each cell, among cells averages, and SEM are shown. The numbers of cells and animals are shown under each plot. I-n Mean amplitudes of AMPAR EPSCs (I), NMDAR EPSC (m), and their ratios (n) at the baseline (base) and the minutes 9-10 after LTP induction (LTP). ${ }^{*} p<0.05,{ }^{* *} p<0.01$; the Wilcoxon matched pairs test in (b, d, f, h, I, $\mathbf{m}, \mathbf{n}$ ); and the one sample $t$-test in $\mathbf{k}$ 
A

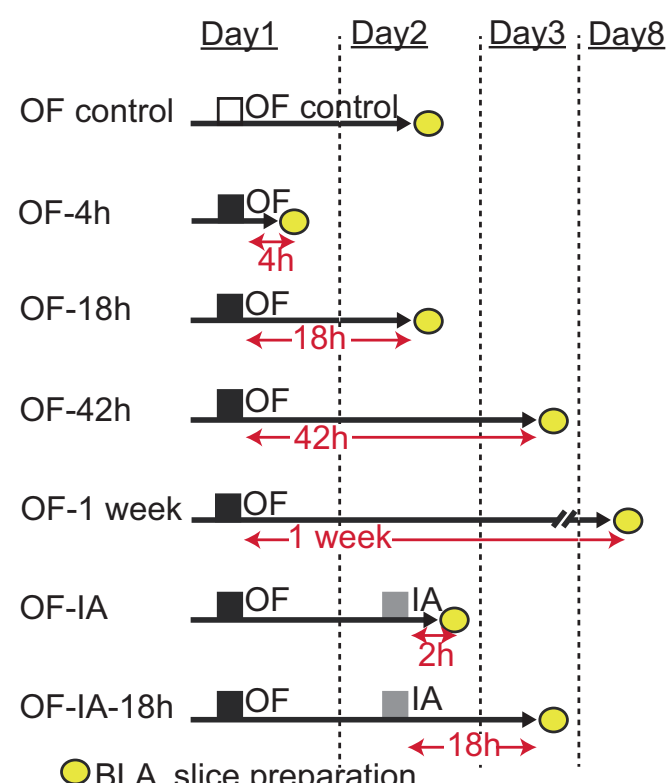

OBLA slice preparation
B
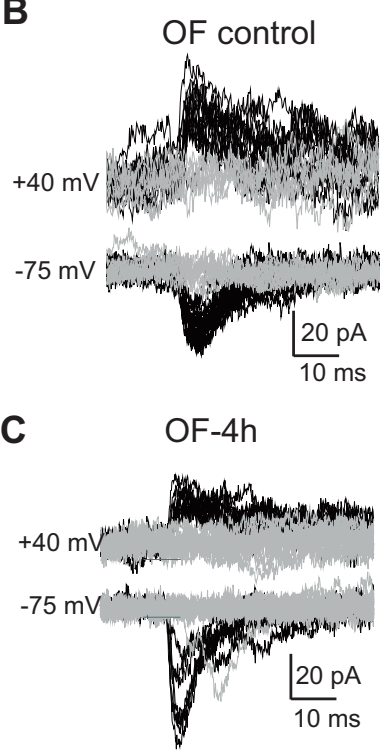

C

$\mathbf{E}$
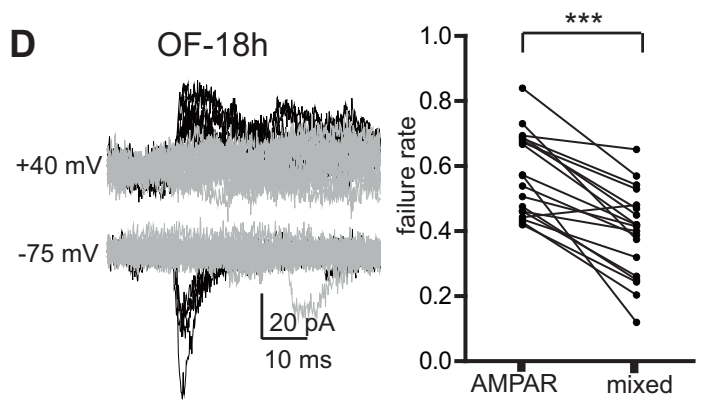

$\mathbf{F}$
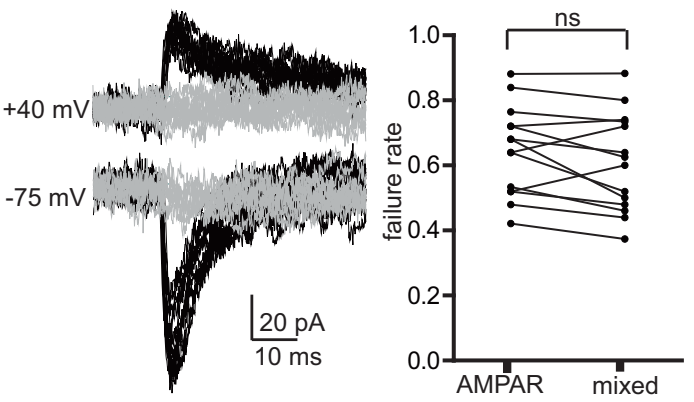

H

OF-IA-18h
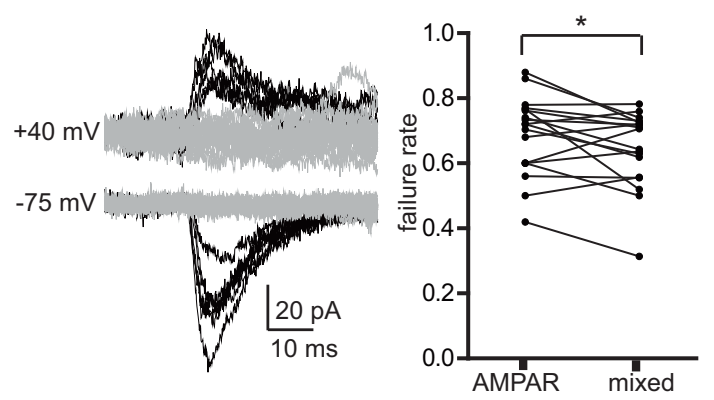
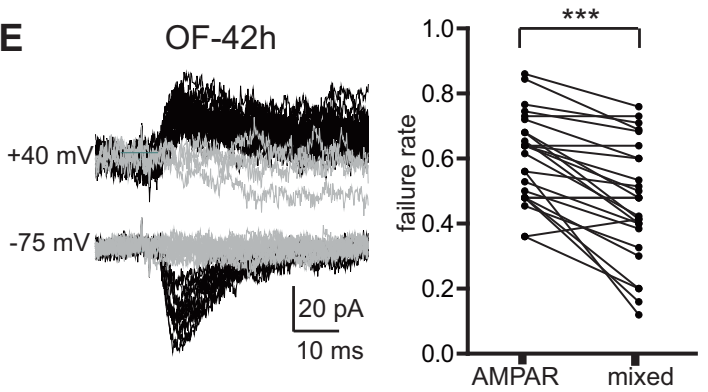

G
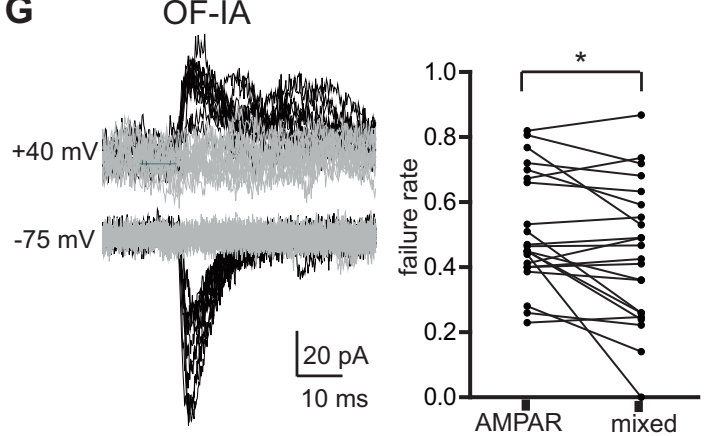

I

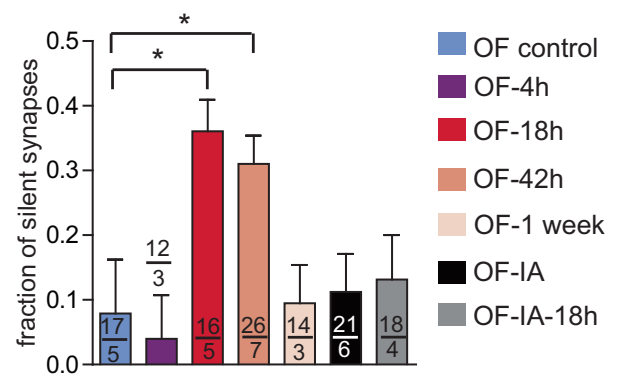

Fig. 3 OF generates silent synapses and IA training eliminates them. a Seven experimental groups. b-h Left: Example traces at -75 (lower) and $+40 \mathrm{mV}$ (upper) to measure AMPAR and AMPAR-NMDAR mixed currents, respectively. Successes EPSC responses and failures are shown respectively in black and gray. Right: Failure rates of AMPAR and AMPAR-NMDAR mixed currents. i Summary data for the fractions of silent synapses. Data in panels (b) and (d) have been published earlier [11] and are shown here for comparison. ${ }^{*} p<0.05, p^{* * *}<0.001 ;$ the paired $t$-test in (b-h), the unpaired $t$-test in (i) 
A

A B<smiles>C=C(C)C(C)CCCC(C)(C)C(C)(C)C</smiles>

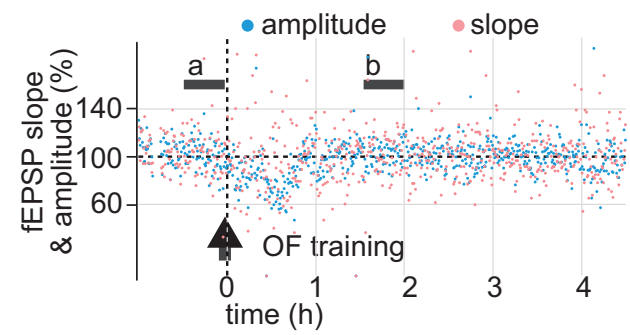

BLA

$\underline{\text { OF control }}$
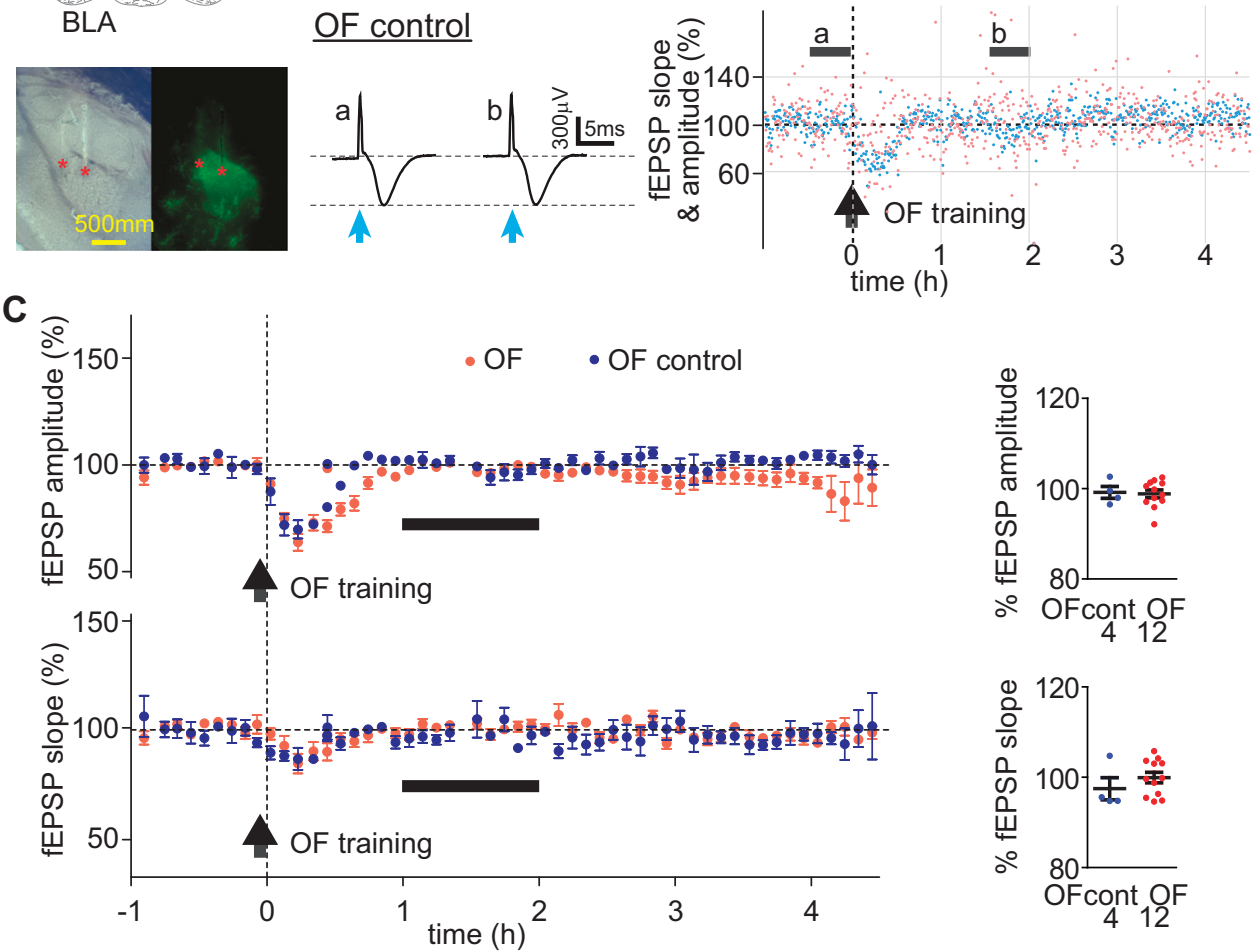

Fig. 4 OF does not cause lasting changes in synaptic efficacy in free moving mice. In vivo recording of light-evoked LFP during OF. a Upper: The schematics represent ChR2-Venus expressing dmPFC neurons projecting to BLA and bilaterally implanted optrodes. Lower: Verification of optrode location. Left: visible light, right: Venus fluorescence images. The positions of electrode tips marked as red stars. $\mathbf{b}$ Left: Examples of fEPSP traces before (a) and after (b) OF training (averaged over the time range shown by horizontal black bars on the right) in an OF (OF) and an OF control mouse (OF control). The vertical light-blue arrows indicate the blue light stimulation. Right: fEPSP amplitudes (light blue) and slopes (pink) normalized to the baseline. Vertical arrows indicate the time of OF training. Time $=0(\mathrm{~h})$ indicates the moment when the subject was returned to the home cage from the OF training chamber. c Left: Summary fEPSP data. Horizontal black bars indicate the time range corresponding to the averaged fEPSP data on the right. Each data point represents the average for 6 min. Right: fEPSP slopes and amplitudes during the hour 2 after OF training for each amygdala and averages. Error bars represent SEMs. The numbers of amygdalae recorded from are shown under each plot

associative learning [34]. The "weak" STDP protocol, consisted of 45 paired pre- and postsynaptic stimuli, induced LTP at dmPFC-BLA synapses in the OF-experienced mice, but not in control mice. Such "low-threshold LTP" was found in slices prepared at 18 and $42 \mathrm{~h}$ after OF. Thus, the OF procedure elicited certain neuronal changes that enabled the low-threshold LTP and lasted for about two days. When OF was followed by IA training one day later, the same protocol no longer induced LTP. It suggests that IA training recruits the OF-enabled plasticity and facilitates the dmPFC-BLA pathway. Such facilitation is, however, difficult to probe by ex vivo methods because of the variability of ChR2 expression, which masks possible differences in synaptic efficacy between different animals. Instead, we performed the within the animal comparisons by in vivo recording, tracking the change in the light-evoked LFPs along the OF-IA behavioral sequence. It revealed lasting synaptic facilitation after IA training in the OF-experienced mice, but not the OF control. Such in vivo facilitation is consistent with the IA occlusion of the low-threshold plasticity ex vivo.
The facilitation of the dmPFC-BLA pathway during IA training appears similar to the earlier described plasticity during associative learning in rats. The examples include facilitation in the thalamic [37] and cortical inputs [35] to the amygdala during fear conditioning and in the Schaffer collateral pathway during inhibitory avoidance training [44]. Meanwhile, the OF-enabled facilitation was distinct in two aspects-first, it was an aberrant circuit response enabled by a prior traumatic experience, and not seen in the control animals; while the above examples involve normal learning in naive animals. Second, most forms of LTP induced in inputs to the amygdala by fear conditioning or various ex vivo protocols are expressed presynaptically [35-41] but see $[45,46]$. In contrast, the OF-enabled LTP did not recruit presynaptic mechanisms, since LTP induction did not decrease the paired-pulse ratio or the CV of postsynaptic responses, and LTP magnitude did not correlate with the relative $\mathrm{CV}^{-2}$ value. This lack of presynaptic expression could not be explained by the ChR2-interference with presynaptic plasticity [43] because a stronger STDP protocol induced a presynaptically expressed LTP 
A
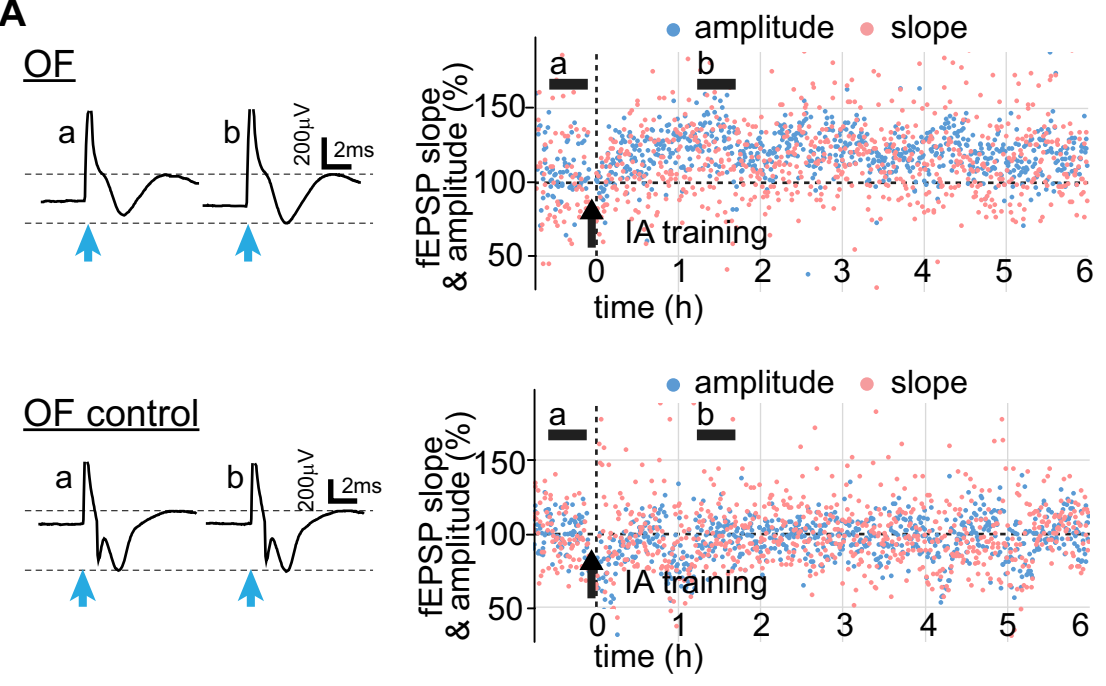

$\mathbf{B}$
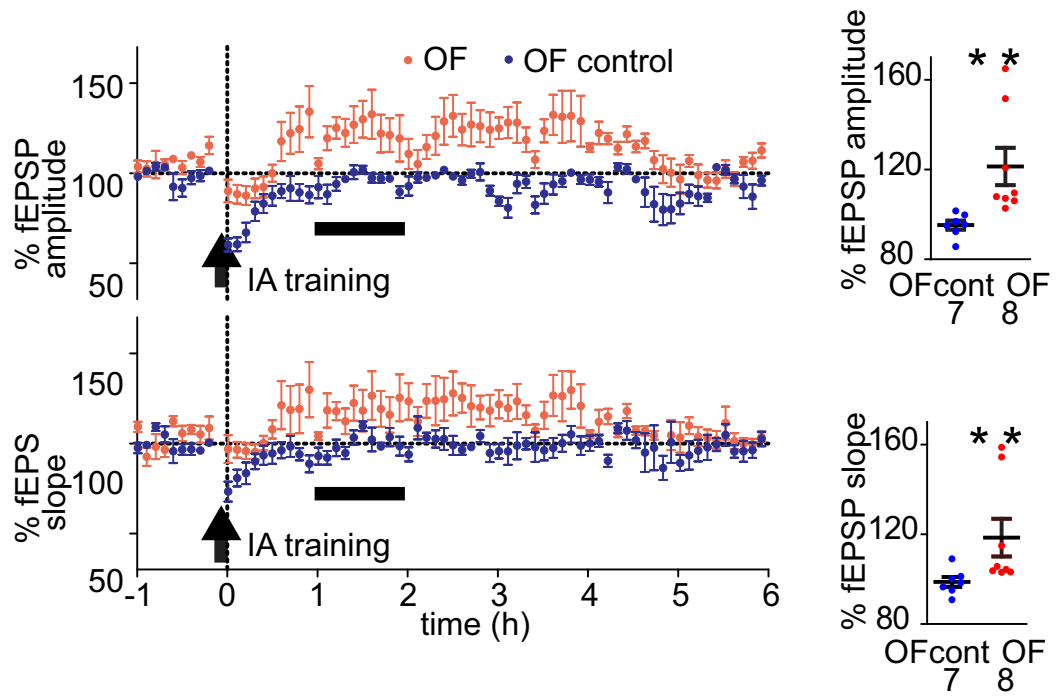

Fig. 5 OF enables facilitation of the dmPFC-BLA pathway by IA training in free moving mice. a Left: Examples of fEPSP traces before (a) and after (b) IA training (averaged over the time range shown by horizontal black bars on the right) in an OF-experienced mouse (OF) and an OF control mouse (OF control). The vertical light-blue arrows indicate the blue light stimulation. Right: fEPSP amplitudes (light blue) and slopes (pink) normalized to the baseline. Vertical arrows indicate the time of IA training. Time $=0(\mathrm{~h})$ indicates the moment when the subject was returned to the home cage, and recording resumed. Recording was interrupted by IA training for about 10 min because the training required disconnection from the recording system. b Summary fEPSP data. Horizontal black bars indicate the time range corresponding to the averaged fEPSP data on the right. Each data point represents the average for $6 \mathrm{~min}$. Right: fEPSP slopes and amplitudes during the hour 2 after OF training for each amygdala and averages. Error bars represent SEMs. The numbers of amygdalae recorded from are shown under each plot. ${ }^{* *} p<0.01$, compared to baseline, Wilcoxon signed rank test

in slices from naive mice despite the presence of ChR2. A plausible explanation is that the "normal" presynaptically expressed LTP has a higher induction threshold than the "aberrant" low-threshold LTP, which had postsynaptic expression.

We could not, however, demonstrate directly the insertion of AMPAR into silent synapses after application of the low-threshold STDP protocol, because of the incompatibility of the internal solution with the simultaneous measurement of the AMPAR and NMDAR currents. Nevertheless, another pairing protocol in the voltage-clamp mode, $2 \mathrm{~Hz}$ presynaptic stimulation paired with continuous postsynaptic depolarization, produced synaptic facilitation in the OF mice, but not in controls, and the facilitation was accompanied by increases in the AMPAR/NMDAR ratios, which indicated that OF indeed enables the postsynaptic plasticity.

Together with the enhancement of LTP induction, OF increased the proportion of silent synapses at 18 and $42 \mathrm{~h}$, but not at $4 \mathrm{~h}$ or one week after OF, indicating their formation and disappearance are slow processes, which require hours and days, respectively. Furthermore, OF procedure did not change the synaptic efficacy in dmPFC-BLA synapses in vivo at least $4 \mathrm{~h}$ after OF. Together with the previous finding that OF increases the NMDAR currents without changing the AMPAR currents evoked by the minimal-like stimulation of the dmPFC-BLA synapses [11], these results suggest that silent synapses formed de novo, rather than via a quicker synaptic process-the removal of AMPA receptors from functional synapses. While the mechanisms of silent synapse formation after OF remain unknown, studies of sensory deprivation during the post-critical period provided evidence that (a) sensory modulation generates silent synapses in the hippocampal CA3-CA1 connections [47] and the thalamocortical pathway [48], and that (b) the process required GluN2B [48]. Moreover, the GluN2B drives synaptogenesis while preventing the membrane incorporation of AMPAR $[49,50]$. These findings suggest that certain patterns of neuronal activity cause the formation of silent synapses in the 
GluN2B-dependent manner. Therefore, we speculate that OF induces unidentified activities in the dmPFC-BLA pathway that trigger the process of generating silent synapses. Our earlier finding that OF decreases AMPA/NMDA ratio in the dmPFC-BLA pathway in a circuit-specific manner, without affecting the TeABLA pathway [11], suggest that silent synapses are formed in specific projections. Although pathway-specific activity would be a plausible underlying mechanism, additional studies are required for proving it.

The co-occurrence of silent synapses and plasticity in dmPFCBLA pathway supports the idea that the OF-generated silent synapses are responsible for the enhanced LTP. First, the presence of silent synapses at 18 and $42 \mathrm{~h}$ after OF coincided with the LTP and the disappearance of silent synapses after IA training was accompanied by the loss of LTP. Second, the OF-enabled LTP was expressed postsynaptically, which is consistent with conversion of silent synapses into functional ones via insertion of AMPAR [18]. Third, the in vivo recordings showed that IA training facilitated evoked-LFPs only in the OF-experienced animals, which had silent synapses, suggesting that silent synapses are needed for the LFP facilitation in vivo. This in vivo facilitation also suggests that IA training converts silent synapses to functional ones and thereby occludes the ex vivo LTP, rather than causing a withdrawal of silent synapses.

These findings, of course, do not rule out entirely a possibility that LTP facilitation occurs via a postsynaptic mechanism independent of silent synapses, for example, via insertion of AMPAR in the existing functional synapses. Furthermore, the LFP recorded in vivo reflect not only the efficacy of glutamatergic synapses but also the excitability of the local neurons and the balance between evoked inhibition and excitation. Therefore, a direct test for the causal role of silent synapses in LTP would require their selective artificial elimination, which remains a technical challenge.

Even without the direct causal proof, the OF-generated silent synapses are the potential factor for the OF-induced synaptic facilitation in dmPFC-BLA pathway ex vivo and, presumably, in vivo. The presence of such abnormal plasticity may represent the time window of vulnerability to stress, which opens within several hours after OF with the formation of silent synapses, lasts for at least two days, and enables synaptic facilitation leading to maladaptive behaviors when the animal encounters another fearful event, like IA training.

Our finding indicated that, in the OF mice, IA training facilitated amygdala responses to the stimulation of the dmPFC axons in vivo for about $4 \mathrm{~h}$, which may reflect a strengthening of functional connectivity between the structures. The 4-hour window after IA training is the critical period when IA memory consolidation can be enhanced by co-activating BLA, prefrontal cortex and several other BLA-connected areas [15-17]. It points to a possibility that the IA training-induced "aberrant" facilitation in the dmPFC-BLA pathway is the cause for the OF-enhanced inhibitory avoidance memories in the previously traumatized mice.

\section{FUNDING AND DISCLOSURE}

The study was supported by the NIH grants MH112093, MH118604, and the Whitehall Foundation.

\section{ADDITIONAL INFORMATION}

Supplementary Information accompanies this paper at (https://doi.org/10.1038/ s41386-019-0342-7)

Competing interests: The authors declare that they have no conflict of interest.

Publisher's note: Springer Nature remains neutral with regard to jurisdictional claims in published maps and institutional affiliations.

\section{REFERENCES}

1. Cougle JR, Resnick H, Kilpatrick DG. Does prior exposure to interpersonal violence increase risk of PTSD following subsequent exposure? Behav Res Ther. 2009;47:1012-7.

2. Eriksson CB, Vende Kemp H, Gorsuch R, Hoke S, Foy DW. Trauma exposure and PTSD symptoms in international relief and development personnel. J Trauma Stress. 2001;14:205-12.

3. Jeon D, Kim S, Chetana M, Jo D, Ruley HE, Lin SY, et al. Observational fear learning involves affective pain system and Cav1.2 Ca2 + channels in ACC. Nat Neurosci. 2010;13:482-8.

4. Chen Q, Panksepp JB, Lahvis GP. Empathy is moderated by genetic background in mice. PLoS ONE 2009;4:e4387.

5. Bruchey AK, Jones CE, Monfils MH. Fear conditioning by-proxy: social transmission of fear during memory retrieval. Behav Brain Res. 2010;214:80-4.

6. Knapska E, Mikosz M, Werka T, Maren S. Social modulation of learning in rats. Learn Mem. 2010;17:35-42.

7. Warren BL, Vialou VF, Iniguez SD, Alcantara LF, Wright KN, Feng J, et al. Neurobiological sequelae of witnessing stressful events in adult mice. Biol Psychiatry. 2013;73:7-14.

8. Iniguez SD, Flores-Ramirez FJ, Riggs LM, Alipio JB, Garcia-Carachure I, Hernandez $M A$, et al. Vicarious social defeat stress induces depression-related outcomes in female mice. Biol Psychiatry. 2018;83:9-17.

9. Keum S, Kim A, Shin JJ, Kim JH, Park J, Shin HS. A missense variant at the Nrxn3 locus enhances empathy fear in the mouse. Neuron. 2018;98:588-601 e5.

10. Allsop SA, Wichmann R, Mills F, Burgos-Robles A, Chang CJ, Felix-Ortiz AC, et al. Corticoamygdala transfer of socially derived information gates observational learning. Cell . 2018;173:1329-42 e18.

11. Ito W, Erisir A, Morozov A. Observation of distressed conspecific as a model of emotional trauma generates silent synapses in the prefrontal-amygdala pathway and enhances fear learning, but ketamine abolishes those effects. Neuropsychopharmacol. 2015;40:2536-45.

12. Zhang $\mathrm{Y}$, Fukushima $\mathrm{H}$, Kida $\mathrm{S}$. Induction and requirement of gene expression in the anterior cingulate cortex and medial prefrontal cortex for the consolidation of inhibitory avoidance memory. Mol Brain. 2011;4:4.

13. Izquierdo LA, Barros DM, da Costa JC, Furini C, Zinn C, Carnmarota M, et al. A link between role of two prefrontal areas in immediate memory and in long-term memory consolidation. Neurobiol Learn Mem. 2007;88:160-66.

14. McGaugh JL. The amygdala modulates the consolidation of memories of emotionally arousing experiences. Annu Rev Neurosci. 2004;27:1-28.

15. McGaugh JL. Memory consolidation and the amygdala: a systems perspective. Trends Neurosci. 2002;25:456.

16. Malin EL, Ibrahim DY, Tu JW, McGaugh JL. Involvement of the rostral anterior cingulate cortex in consolidation of inhibitory avoidance memory: interaction with the basolateral amygdala. Neurobiol Learn Mem. 2007;87:295-302.

17. Malin EL, McGaugh JL. Differential involvement of the hippocampus, anterior cingulate cortex, and basolateral amygdala in memory for context and footshock. Proc Natl Acad Sci USA. 2006;103:1959-63.

18. Hanse $E$, Seth $H$, Riebe I. AMPA-silent synapses in brain development and pathology. Nat Rev Neurosci. 2013;14:839-50.

19. Ma YY, Lee BR, Wang X, Guo C, Liu L, Cui R, et al. Bidirectional modulation of incubation of cocaine craving by silent synapse-based remodeling of prefrontal cortex to accumbens projections. Neuron. 2014;83:1453-67.

20. Brown TE, Lee BR, Mu P, Ferguson D, Dietz D, Ohnishi YN, et al. A silent synapsebased mechanism for cocaine-induced locomotor sensitization. J Neurosci. 2011;31:8163-74.

21. Lee $B R, M a Y Y$, Huang $Y H$, Wang $X$, Otaka M, Ishikawa $M$, et al. Maturation of silent synapses in amygdala-accumbens projection contributes to incubation of cocaine craving. Nat Neurosci. 2013;16:1644-51.

22. Liao D, Hessler NA, Malinow R. Activation of postsynaptically silent synapses during pairing-induced LTP in CA1 region of hippocampal slice. Nature. 1995;375:400-4.

23. Lisman J, Raghavachari S. A unified model of the presynaptic and postsynaptic changes during LTP at CA1 synapses. Science's STKE. 2006;2006:re11.

24. El-Ghundi M, O'Dowd BF, George SR. Prolonged fear responses in mice lacking dopamine D1 receptor. Brain Res. 2001;892:86-93.

25. Petreanu L, Mao T, Sternson SM, Svoboda K. The subcellular organization of neocortical excitatory connections. Nature. 2009;457:1142-5.

26. Morozov A, Sukato $D$, Ito W. Selective suppression of plasticity in amygdala inputs from temporal association cortex by the external capsule. J Neurosci. 2011;31:339-45.

27. Daw Ml, Tricoire L, Erdelyi F, Szabo G, McBain CJ. Asynchronous transmitter release from cholecystokinin-containing inhibitory interneurons is widespread and target-cell independent. J Neurosci. 2009;29:11112-22.

28. McDonald AJ. Neurons of the lateral and basolateral amygdaloid nuclei: a Golgi study in the rat. J Comp Neurol. 1982;212:293-312. 
29. Malinow R, Tsien RW. Presynaptic enhancement shown by whole-cell recordings of long-term potentiation in hippocampal slices. Nature. 1990;346:177-80.

30. Marie H, Morishita W, Yu X, Calakos N, Malenka RC. Generation of silent synapses by acute in vivo expression of CaMKIV and CREB. Neuron. 2005;45:741-52.

31. Pan BX, Ito W, Morozov A. Divergence between thalamic and cortical inputs to lateral amygdala during juvenile-adult transition in mice. Biol Psychiatry. 2009;66:964-71.

32. Kullmann DM. Amplitude fluctuations of dual-component EPSCs in hippocampal pyramidal cells: implications for long-term potentiation. Neuron. 1994;12: 1111-20.

33. Huang $Y H$, Lin $Y, M u ~ P$, Lee BR, Brown TE, Wayman $G$, et al. In vivo cocaine experience generates silent synapses. Neuron. 2009;63:40-7.

34. Sjostrom PJ, Turrigiano GG, Nelson SB. Rate, timing, and cooperativity jointly determine cortical synaptic plasticity. Neuron. 2001;32:1149-64.

35. Tsvetkov E, Carlezon WA, Benes FM, Kandel ER, Bolshakov VY. Fear conditioning occludes LTP-induced presynaptic enhancement of synaptic transmission in the cortical pathway to the lateral amygdala. Neuron. 2002;34:289-300.

36. Huang YY, Kandel ER. Postsynaptic induction and PKA-dependent expression of LTP in the lateral amygdala. Neuron. 1998;21:169-78.

37. McKernan MG, Shinnick-Gallagher P. Fear conditioning induces a lasting potentiation of synaptic currents in vitro. Nature. 1997;390:607-11.

38. Pan BX, Vautier F, Ito W, Bolshakov VY, Morozov A. Enhanced corticoamygdala efficacy and suppressed fear in absence of Rap1. J Neurosci. 2008;28:2089-98.

39. Humeau Y, Shaban H, Bissiere S, Luthi A. Presynaptic induction of heterosynaptic associative plasticity in the mammalian brain. Nature. 2003;426:841-5.
40. Shaban H, Humeau Y, Herry C, Cassasus G, Shigemoto R, Ciocchi S, et al. Generalization of amygdala LTP and conditioned fear in the absence of presynaptic inhibition. Nat Neurosci. 2006;9:1028-35.

41. Fourcaudot $E$, Gambino $F$, Humeau $Y$, Casassus $G$, Shaban $H$, Poulain B, et al CAMP/PKA signaling and RIM1alpha mediate presynaptic LTP in the lateral amygdala. Proc Natl Acad Sci USA. 2008;105:15130-5.

42. Zucker RS, Regehr WG. Short-term synaptic plasticity. Annu Rev Physiol. 2002;64:355-405.

43. Zhang YP, Oertner TG. Optical induction of synaptic plasticity using a lightsensitive channel. Nat Methods. 2007;4:139-41.

44. Whitlock JR, Heynen AJ, Shuler MG, Bear MF. Learning induces long-term potentiation in the hippocampus. Science. 2006;313:1093-7.

45. Rumpel S, LeDoux J, Zador A, Malinow R. Postsynaptic receptor trafficking underlying a form of associative learning. Science. 2005;308:83-8.

46. Nabavi S, Fox R, Proulx CD, Lin JY, Tsien RY, Malinow R. Engineering a memory with LTD and LTP. Nature. 2014;511:348-52.

47. Milshtein-Parush $\mathrm{H}$, Frere $\mathrm{S}$, Regev L, Lahav C, Benbenishty A, Ben-Eliyahu S, et al. Sensory deprivation triggers synaptic and intrinsic plasticity in the hippocampus. Cereb Cortex. 2017;27:3457-70.

48. Chung S, Jeong JH, Ko S, Yu X, Kim YH, Isaac JTR, et al. Peripheral sensory deprivation restores critical-period-like plasticity to adult somatosensory thalamocortical inputs. Cell Rep. 2017;19:2707-17.

49. Gambrill AC, Barria A. NMDA receptor subunit composition controls synaptogenesis and synapse stabilization. Proc Natl Acad Sci USA. 2011;108:5855-60.

50. Hall BJ, Ripley B, Ghosh A. NR2B signaling regulates the development of synaptic AMPA receptor current. The. J Neurosci: Off J Soc Neurosci. 2007;27:13446-56. 